\title{
COMPARISON OF EIGENVALUES ASSOCIATED WITH LINEAR DIFFERENTIAL EQUATIONS OF ARBITRARY ORDER
}

BY

R. D. GENTRY( $\left.{ }^{1}\right)$ AND C. C. TRAVIS

ABSTRACT. Existence and comparison theorems for eigenvalues of $(k, n-k)$-focal point and $(k, n-k)$-conjugate point problems are proved for a class of $n$th order linear differential equations for arbitrary $n$.

1. Introduction. We shall be interested in establishing existence and comparison theorems for the smallest positive eigenvalues of a class of $n$th order nonselfadjoint $(k, n-k)$-focal point eigenvalue problems. In particular, we shall consider the differential equations

$$
\begin{array}{r}
{\left[a(x) u^{(k)}(x)\right]^{(n-k)}-(-1)^{n-k} \lambda \sum_{i=0}^{k-1} p_{i}(x) u^{(i)}(x)=0} \\
u(\alpha)=u^{\prime}(\alpha)=\cdots=u^{(k-1)}(\alpha)=0, \\
u_{1}(\beta)=u_{1}^{\prime}(\beta)=\cdots=u_{1}^{(n-k-1)}(\beta)=0,
\end{array}
$$

and

$$
\begin{array}{r}
{\left[A(x) \nu^{(k)}(x)\right]^{(n-k)}-(-1)^{n-k} \Lambda \sum_{i=0}^{k-1} P_{i}(x) \nu^{(i)}(x)=0,} \\
\nu(\alpha)=\nu^{\prime}(\alpha)=\cdots=\nu^{(k-1)}(\alpha)=0, \\
\nu_{1}(\beta)=\nu_{1}^{\prime}(\beta)=\cdots=\nu_{1}^{(n-k-1)}(\beta)=0,
\end{array}
$$

where $k=1,2, \ldots, n-1$, and $u_{1}(x)$ and $\nu_{1}(x)$ are defined by

$$
u_{1}(x)=a(x) u^{(k)}(x) \text { and } v_{1}(x)=A(x) \nu^{(k)}(x)
$$

Received by the editors March 19, 1975.

AMS (MOS) subject classifications (1970). Primary 34B05; Secondary 34C10.

Key words and phrases. Eigenvalue, linear differential equations of arbitrary order, eigenvalue comparison, focal point, $u_{0}$-positive, positive cone.

$\left({ }^{1}\right)$ This work was partially supported by the National Research Council of Canada under Grant A9100. 
Throughout this paper we shall assume that the functions $a(x)$ and $A(x)$ are positive and of class $C^{(n-k)}[\alpha, \beta]$ and that the functions $p_{i}(x)$ and $P_{i}(x)$ are of class $C[\alpha, \beta]$ for each $i=0,1,2, \ldots, k-1$ with at least one function $P_{i}(x)$ and one function $P_{i}(x)$ not identically zero on $[\alpha, \beta]$

A survey of the classical comparison theorems for selfadjoint eigenvalue problems of order $2 n$ may be found in the book of C. A. Swanson [4]. Our results will generalize these classical selfadjoint comparison theorems in two distinct ways. First we shall allow the principal part of the differential equation to be of arbitrary order; thereby allowing the problem to be nonselfadjoint. Second, even in the case where the principal part of the differential equation is formally selfadjoint; that is when $n=2 k$, the eigenvalue problem (1.1) does not reduce to a selfadjoint eigenvalue problem unless $p_{i}(x)=0$ for $i=1,2, \ldots, k-1$. Only in this case does the Courant minimum principle apply yielding that the least positive eigenvalues of (1.1) and (1.2) satisfy $\Lambda \leqslant \lambda$, if $0<A(x)<a(x)$ and $0 \leqslant$ $p_{0}(x)<P_{0}(x)$ on $[\alpha, \beta]$.

The method that we employ to establish existence and comparison theorems for the $(k, n-k)$-focal point problems (1.1) and (1.2) can also be used to establish existence and comparison theorems for the $(k, n-k)$-conjugate point eigenvalue problem. That is, equations (1.1) and (1.2) subject to the boundary conditions

$$
\begin{aligned}
& u(\alpha)=u^{\prime}(\alpha)=\cdots=u^{(k-1)}(\alpha)=0, \\
& u(\beta)=u^{\prime}(\beta)=\cdots=u^{(n-k-1)}(\beta)=0,
\end{aligned}
$$

and

$$
\nu(\alpha)=\nu^{\prime}(\alpha)=\cdots=\nu^{(k-1)}(\alpha)=0
$$

$$
\nu(\beta)=\nu^{\prime}(\beta)=\cdots=\nu^{(n-k-1)}(\beta)=0,
$$

respectively. We will state existence and comparison theorems for the $(k, n-k)$ conjugate point eigenvalue problem; however the proofs shall be omitted since they are similar to the focal point case. The main reason for restricting our attention to the $(k, n-k)$-focal point eigenvalue problem is that for this class of problems it is possible to establish eigenvalue comparison theorems of the "integral type", such as were first established by Z. Nehari [3] for second order equations and later extended to selfadjoint equations of order $2 n$ by C. C. Travis [5]. The following are an example of the kind of results we have obtained:

TheOREM 1.1. Assume that $0 \leqslant \int_{x}^{\beta} p_{i}(s) d s$ on $[\alpha, \beta]$ for $i=0,1, \ldots$, $k-1\left(0 \leqslant p_{i}(x)\right.$ on $[\alpha, \beta]$ for $i=0,1, \ldots, k-1$, respectively), then the 
$(k, n-k)$-focal point eigenvalue problem $(1.1)$ (the $(k, n-k)$-conjugate point eigenvalue problem (1.1)-(1.1a), respectively) has at least one real eigenvalue which is positive and smaller than the absolute value of any other eigenvalue. The eigenfunction associated with this eigenvalue is positive on $(\alpha, \beta)$.

THEOREM 1.2. If

(ii) $\quad \int_{x}^{\beta} p_{i}(s) d s \leqslant \int_{x}^{\beta} P_{i}(s) d s$ on $[\alpha, \beta]$ for $i=0,1, \ldots, k-1$,

(iii) $\quad \int_{\alpha}^{x} \frac{1}{a(s)} d s \leqslant \int_{\alpha}^{x} \frac{1}{A(s)} d s$ on $[\alpha, \beta]$,

then the smallest positive eigenvalues $\lambda_{0}$ and $\Lambda_{0}$, of the $(k, n-k)$-focal point eigenvalue problems (1.1) and (1.2) respectively, satisfy $\Lambda_{0} \leqslant \lambda_{0}$ with equality if and only if $a(x) \equiv A(x)$ and $p_{i}(x) \equiv P_{i}(x)$ on $[\alpha, \beta]$ for $i=0,1, \ldots, k-1$.

THEOREM 1.3. If

$$
\begin{aligned}
& 0 \leqslant p_{i}(x) \leqslant P_{i}(x) \text { on }[\alpha, \beta] \text { for } i=0,1, \ldots, k-1, \\
& 0<A(x) \leqslant a(x) \text { on }[\alpha, \beta],
\end{aligned}
$$

then the smallest positive eigenvalues, $\lambda_{0}$ and $\Lambda_{0}$, of the $(k, n-k)$-conjugate point eigenvalue problems (1.1)-(1.1a) and (1.2)-(1.2a) respectively, satisfy $\Lambda_{0}$ $\leqslant \lambda_{0}$ with equality if and only if $a(x) \equiv A(x)$ and $p_{i}(x) \equiv P_{i}(x)$ on $[\alpha, \beta]$ for $i=$ $0,1, \ldots, k-1$.

In $\$ 2$ we introduce some basic results concerning eigenvalues of operators defined on a Banach space $B$ which are $u_{0}$-positive with respect to a cone $P$. We introduce in $\$ 3$ integral equations equivalent to the differential equations of (1.1) and (1.2) and establish some results concerning the $u_{0}$-positivity and ordering of their associated operators. Combining these results in $\$ 4$ we prove our main result and provide some applications of these results.

2. $u_{0}$-positive operators. The theory and application of $u_{0}$-positive operators has been extensively developed by Krasnosel'skii in his book [1]. It will suffice for our applications to consider a real Banach space $B$.

Definition 2.1. A closed subset $P$ of $B$ is called a cone if it satisfies

$$
u \in P \text { and } \nu \in P \rightarrow u+\nu \in P
$$

$$
u \in P \text { and } \tau \geqslant 0 \rightarrow \tau u \in P
$$

$$
u \in P \text { and }-u \in P \rightarrow u=0 .
$$


Each "nonnegative" cone $P$ in a Banach space $B$ generates a partial ordering on both the space $B$ and the space of operators mapping $B$ into itself.

Definition 2.2. For a given cone $P$ of a Banach space $B$ we say that

(i) for elements $u, v \in B, u \leqslant v$ if and only if $v-u \in P$,

(ii) for operators $M$ and $L$ defined on $B, M \leqslant L$ with respect to $P$ if $M u$ $\leqslant L u$ for all $u \in P$.

Definition 2.3. A bounded linear operator $M$ defined on a Banach space $B$ is $u_{0}$-positive (with respect to a cone $P$ of $B$ ) if there exists a nonzero element $u_{0} \in P$ such that for every nonzero element $u \in P$ there exist positive numbers $k_{1}$ and $k_{2}$ with $k_{1} u_{0} \leqslant M u \leqslant k_{2} u_{0}$.

We next introduce two important theorems, concerning $u_{0}$-positive operators. The first is a result of M. A. Krasnosel'skii [1, pp. 67-81] which will enable us to assert the existence of a least positive eigenvalue for the $(k, n-k)$ focal point eigenvalue problems (1.1) and (1.2). The second is a result of $C$. $C$. Travis [5, pp. 365] which will be fundamental in establishing comparison theorems for eigenvalues.

THEOREM 2.1. If $L$ is a compact $u_{0}$-positive operator with respect to the cone $P$, then $L$ has exactly one (normalized) eigenvector in $P$ and the corresponding eigenvalue is positive and larger than the absolute value of any other eigenvalue.

THEOREM 2.2. Let $L$ and $M$ be linear operators and assume that at least one of the operators is $u_{0}$-positive. If $L \leqslant M$ and

$$
L u_{1}=\lambda_{1} u_{1} \quad\left(u_{1} \in P, \lambda_{1}>0\right)
$$

and

$$
M u_{2}=\lambda_{2} u_{2} \quad\left(u_{2} \in P, \lambda_{2}>0\right)
$$

then $\lambda_{1} \leqslant \lambda_{2}$, and if $\lambda_{1}=\lambda_{2}, u_{1}$ is a scalar multiple of $u_{2}$.

3. $u_{0}$-positive operators. For a given positive function $b(x) \in C^{(n-k)}[\alpha, \beta]$ we define the differential operator $D$ by

$$
\begin{gathered}
D[u]=(-1)^{n-k}\left[b(x) u^{k}(x)\right]^{(n-k)}, \\
u(\alpha)=u^{\prime}(\alpha)=\cdots=u^{k-1}(\alpha)=0, \\
u_{1}(\beta)=u_{1}^{\prime}(\beta)=\cdots=u_{1}^{(n-k-1)}(\beta)=0 .
\end{gathered}
$$

LEMMA 3.1. The Green's function $G(x, s)$ for the operator $(3.1)$ is given by 
(3.2) $G(x, s)=\left\{\begin{array}{r}\frac{1}{(n-k-1) !} \int_{\alpha}^{x} \int_{\alpha}^{x_{k}} \cdots \int_{\alpha}^{x_{2}} \frac{\left(s-x_{1}\right)^{n-k-1}}{b\left(x_{1}\right)} d x_{1} \cdots d x_{k} \\ \text { for } \alpha \leqslant x \leqslant s \leqslant \beta, \\ \frac{1}{(k-1) !} \int_{\alpha}^{s} \int_{\alpha}^{x_{n-k}} \cdots \int_{\alpha}^{x_{2}} \frac{\left(x-x_{1}\right)^{k-1}}{b\left(x_{1}\right)} d x_{1} \cdots d x_{n-k} \\ \quad \text { for } \alpha \leqslant s \leqslant x \leqslant \beta .\end{array}\right.$

The verification of Lemma 3.1 is straightforward, as the Green's function is uniquely determined by the associated boundary conditions and the continuity and jump conditions at $x=s$. In later applications we will find it convenient to express (3.2) in a more compact form. Applying the Liouville transform to the iterated integrals of (3.2) we obtain

$$
G(x, s)=\frac{1}{(n-k-1) !(k-1) !} \int_{\alpha}^{\delta} \frac{(x-t)^{k-1}(s-t)^{n-k-1}}{b(t)} d t
$$

where $\delta=\operatorname{minimum}\{x, s\}$.

Rather than examining the differential operators implicit in (1.1) and (1.2) we introduce equivalent integral operators defined on the Banach space $B$ of all functions $v$ such that $v^{(k+1)}$ is continuous on $[\alpha, \beta]$ and $v$ satisfies the initial condition $v^{(i)}(\alpha)=0, i=0,1, \ldots, k$ with norm

$$
\|v\|=\max \left\{|v(x)|,\left|v^{\prime}(x)\right|, \ldots, \mid v^{(k+1)}(x) \| x \in[\alpha, \beta]\right\}
$$

Denoting the Green's function (3.2) by $G_{a}(x, s)$ and $G_{A}(x, s)$ when the function $b(x)$ equals $a(x)$ and $A(x)$ respectively, we define the following integral operators on the Banach space $B$.

$$
\begin{aligned}
& M[u]=\int_{\alpha}^{\beta} G_{a}(x, s)\left[\sum_{i=0}^{k-1} p_{i}(s) u^{(i)}(s)\right] d s, \\
& N[u]=\int_{\alpha}^{\beta} G_{A}(x, s)\left[\sum_{i=0}^{k-1} P_{i}(s) u^{(i)}(s)\right] d s, \\
& R[u]=\int_{\alpha}^{\beta} G_{A}(x, s)\left[\sum_{i=0}^{k-1} p_{i}(s) u^{(i)}(s)\right] d s .
\end{aligned}
$$

We shall consider the $u_{0}$-positivity and relationship of these operators with respect to two cones defined in the space $B$ by:

$$
\begin{aligned}
& P_{1}=\left\{v \in B \mid v^{(i)}(x) \geqslant 0 \text { on }[\alpha, \beta] \text { for } i=0,1, \ldots, k-1\right\} ; \\
& P_{2}=\left\{v \in B \mid v^{(i)}(x) \geqslant 0 \text { on }[\alpha, \beta] \text { for } i=0,1, \ldots, k\right\} .
\end{aligned}
$$


LEMMA 3.2. If $0 \leqslant P_{i}(x)$ on $[\alpha, \beta]$ for $i=0,1, \ldots, k-1$ then the operator (3.4) is $u_{0}$-positive with respect to the cone $P_{1}$.

Proof. We define the function $u_{0} \in P_{1}$ by

$$
u_{0}(x)=\int_{\alpha}^{\beta} G_{a}(x, s)\left[\sum_{i=0}^{k-1} p_{i}(s)\right] d s .
$$

To show that the operator $M$ is $u_{0}$-positive let $u$ be an element of $P_{1}$. For convenience we denote the function $M[u](x)$ by $h(x)$. It will suffice to show that there exist positive constants $r_{1}$ and $r_{2}$ such that

$$
r_{1} u_{0}^{(j)}(x) \leqslant h^{(j)}(x) \leqslant r_{2} u_{0}^{(j)}(x), \quad j=0,1, \ldots, k-1 .
$$

Using the (3.3) form of the Green's function it easily follows that

$$
\begin{aligned}
& u_{0}^{(k)}(x)=\frac{1}{a(x)(n-k-1) !} \int_{x}^{\beta}(s-x)^{n-k-1}\left[\sum_{i=0}^{k-1} p_{i}(s)\right] d s, \\
& h^{(k)}(x)=\frac{1}{a(x)(n-k-1) !} \int_{x}^{\beta}(s-x)^{n-k-1}\left[\sum_{i=0}^{k-1} p_{i}(s) u^{(i)}(s)\right] d s .
\end{aligned}
$$

Furthermore, we note the initial condition

$$
\begin{aligned}
u_{0}(\alpha) & =u_{0}^{\prime}(\alpha)=\cdots=u_{0}^{(k-1)}(\alpha)=0, & u_{0}^{(k)}(\alpha)>0 \\
h(\alpha) & =h^{\prime}(\alpha)=\cdots=h^{(k-1)}(\alpha)=0, & h^{(k)}(\alpha)>0 .
\end{aligned}
$$

As $u_{0}^{(k)}$ and $h^{(k)}$ are continuous we may determine positive constants $\epsilon_{1}, \epsilon_{2}$ and a $\xi \in[\alpha, \beta]$ such that for $x \in[\alpha, \xi]$

$$
\epsilon_{1} u_{0}^{(k)}(x) \leqslant h^{(k)}(x) \leqslant \epsilon_{2} u_{0}^{(k)}(x) .
$$

Integrating this inequality using the initial conditions yields the inequalities for $x \in[\alpha, \xi]$,

$$
\epsilon_{1} u_{0}^{(k-1)}(x) \leqslant h^{(k-1)}(x) \leqslant \epsilon_{2} u_{0}^{(k-1)}(x) .
$$

Then, from the fact that $u_{0}^{(k)}(x) \geqslant 0$ and $h^{(k)}(x) \geqslant 0$ on $[\alpha, \beta]$ and the condition (3.8) we may conclude that $u_{0}^{(k-1)}(x)$ and $h^{(k-1)}(x)$ are strictly positive and bounded on $[\xi, \beta]$. Consequently, we may determine positive constants $\delta_{1}, \delta_{2}$ such that for $x \in[\xi, \beta]$

$$
\delta_{1} u_{0}^{(k-1)}(x) \leqslant h^{(k-1)}(x) \leqslant \delta_{2} u_{0}^{(k-1)}(x) .
$$

Setting $r_{1}=\operatorname{maximum}\left\{\epsilon_{1}, \delta_{1}\right\}$ and $r_{2}=\operatorname{maximum}\left\{\epsilon_{2}, \delta_{2}\right\}$ and integrating with the conditions (3.8) the equation (3.7) follows and the lemma is proved.

In the case of selfadjoint eigenvalue problems, considerable interest has been given to establishing eigenvalue comparison theorems in which the lower order coefficients are not necessarily positive and pointwise comparable, but are positive and comparable in a more general integral sense. However, relaxing the condition that the functions $p_{i}$ be positive in favour of the conditions 
$\int_{x}^{\beta} p_{i}(s) d s>0$ we no longer have $u_{0}$-positivity with respect to the cone $P_{1}$. The proof of Lemma 3.2 fails to hold in this case because we may no longer argue the $u^{(k)}(x) \geqslant 0$ and $h^{(k)}(x) \geqslant 0$ on $[\alpha, \beta]$. To circumvent this difficulty we restrict ourselves to the narrower cone $P_{2}$.

LEMMA 3.3. If $0 \leqslant \int_{x}^{\beta} p_{i}(s) d s$ on $[\alpha, \beta]$ for $i=0,1, \ldots, k-1$ then the operator (3.4) is $u_{0}$-positive with respect to the cone $P_{2}$.

Proof. To prove this lemma we proceed exactly as in the proof of Lemma 3.2, except that we consider the function $u \in P_{2}$. To utilize the integral conditions on the functions $p_{i}(x)$ we integrate by parts to obtain the inequality

$$
h^{(k)}(x)=\frac{1}{a(x)(n-k-1) !} \int_{x}^{\beta} \sum_{i=0}^{k-1} \Phi_{i}(x, s) \int_{s}^{\beta} p_{i}(t) d t d s>0
$$

where

$$
\Phi_{i}(x, s)=\frac{d}{d s}(s-x)^{n-k-1} u^{(i)}(s) .
$$

By the same procedure it follows that $u_{0}^{(k)}(x)>0$ on $[\alpha, \beta]$ and consequently we can find positive constants $k_{1}$ and $k_{2}$ such that

$$
k_{i} u_{0}^{(k)}(x) \leqslant h^{(k)}(x)<k_{2} u_{0}^{(k)}(x) \text { on }[\alpha, \beta] .
$$

Integrating using the initial conditions (3.8) provides the necessary inequalities to complete the proof.

LEMMA 3.4. If $\int_{\alpha}^{x}(1 / \alpha(t)) d t \leqslant \int_{\alpha}^{x}(1 / A(t)) d t$ on $[\alpha, \beta]$ then $M \leqslant R$ with respect to the cone $P_{1}$.

Proof. Let $u \in P_{1}$. Then using the (3.3) form of the Green's function we have for each $j=0,1, \ldots, k-1$

$$
\begin{aligned}
M[u]^{(i)}= & \frac{d^{i}}{d x^{j}} M[u](x) \\
= & \int_{\alpha}^{\beta} \sum_{i=0}^{k-1}\left\{\int_{\alpha}^{\delta} \psi_{j}(x, s, t) \frac{1}{a(t)} d t\right\}\left\{p_{i}(s) u^{(i)}(s)\right\} d s \\
= & \int_{\alpha}^{\beta} \sum_{i=0}^{k-1}\left\{\int_{\alpha}^{\delta} \frac{1}{a(t)} d t \psi_{j}(x, s, \delta)\right. \\
& \left.\quad-\int_{\alpha}^{\delta} \int_{\alpha}^{t} \frac{1}{a(r)} d r \frac{\partial}{\partial t} \psi_{j}(x, s, t) d t\right\}\left\{p_{i}(s) u^{(i)}(s)\right\} d s \\
& <\int_{\alpha}^{\beta} \sum_{i=0}^{k-1}\left\{\int_{\alpha}^{\delta} \frac{1}{A(t)} d t \psi_{j}(x, s, \delta)\right. \\
& \left.\quad-\int_{\alpha}^{\delta} \int_{\alpha}^{t} \frac{1}{A(r)} d r \frac{\partial}{\partial t} \psi_{j}(x, s, t) d t\right\}\left\{p_{i}(s) u^{(i)}(s)\right\} d s \\
= & R[u]^{(j)}(x) .
\end{aligned}
$$


Here the function $\psi_{j}(x, s, t)$ is implicitly defined by the first equality. The inequality results from the hypothesis and the observation that $\psi_{j}(x, s, \delta) \geqslant 0$ and $\partial \psi_{j}(x, s, t) / \partial t \leqslant 0$ on the region of integration. Consequently $M[u] \leqslant R[u]$ with respect to the cone $P_{1}$.

LEMMA 3.5. If $\int_{x}^{\beta} p_{i}(s) d s \leqslant \int_{x}^{\beta} P_{i}(s) d s$ on $[\alpha, \beta]$ then $R \leqslant N$ with respect to the cone $P_{2}$.

Proof. Let $u \in P_{2}$ and $\Phi_{i}(x, s)=(d / d s)(s-x)^{n-k-1} u^{(i)}(s)$. Then integrating as we did in (3.9) we see that

$$
\begin{aligned}
R[u]^{(k)}(x) & =\frac{1}{A(x)(n-k-1) !} \int_{x}^{\beta} \sum_{i=0}^{k-1} \Phi_{i}(x, s) \int_{s}^{\beta} p_{i}(r) d r d s \\
& \leqslant \frac{1}{A(x)(n-k-1) !} \int_{x}^{\beta} \sum_{i=0}^{k-1} \Phi_{i}(x, s) \int_{s}^{\beta} P_{i}(r) d r d s \\
& =N[u]^{(k)}(x) .
\end{aligned}
$$

Using the initial conditions $R[u]^{(j)}(\alpha)=0, N[u]^{(j)}(\alpha)=0$ for $j=0,1, \ldots$, $k-1$ and integrating we have the inequality

$$
R[u]^{(j)}(x) \leqslant N[u]^{(j)}(x) \text { for } j=0,1, \ldots, k,
$$

and the conclusion follows.

LEMMA 3.6. If $0<A(x) \leqslant a(x)$ and $\int_{x}^{\beta} p_{i}(s) d s \leqslant \int_{x}^{\beta} P_{i}(s) d s$ for $i=0,1$, $\ldots, k-1$ then $M \leqslant N$ with respect to the cone $P_{2}$.

Proof. The proof consists of showing that $M \leqslant R$ with respect to $P_{2}$ employing the same ideas used to prove the previous lemmas. Then using the transitivity of the partial order $\leqslant$ the conclusion follows. We will omit the details.

LEMMA 3.7. The operator $M$ given by (3.4) is completely continuous on the Banach space $B$.

Proof. Since $p_{i} \in C[\alpha, \beta]$ and $a(x) \in C^{n-k}[\alpha, \beta]$ the kernel of the $k$ th derivative of $M[u](x)$ (see the proof of Lemma 3.2) is continuous on $[\alpha, \beta]$ and hence the operator $M$ may be shown to be equicontinuous and uniformily bounded on any bounded subset of $B$. Consequently from Ascoli's Theorem we conclude that $M$ is a compact operator on $B$.

4. Comparison theorem. Our main result is given by the

THEOREM 4.1. Let the coefficients of the eigenvalue problems (1.1) and (1.2) satisfy the conditions

$$
0 \leqslant p_{i}(x) \text { for } i=0,1, \ldots, k-1 \text { on }[\alpha, \beta],
$$




$$
\begin{gathered}
\int_{x}^{\beta} p_{i}(s) d s \leqslant \int_{x}^{\beta} P_{i}(s) d s \quad \text { for } i=0,1, \ldots, k-1 \text { on }[\alpha, \beta], \\
\int_{\alpha}^{x} \frac{1}{a(s)} d s \leqslant \int_{\alpha}^{x} \frac{1}{A(s)} d s \text { on }[\alpha, \beta] .
\end{gathered}
$$

Then

(i) there exist least positive eigenvalues $\lambda_{0}$ of (1.1) and $\Lambda_{0}$ of (1.2),

(ii) $\Lambda_{0} \leqslant \lambda_{0}$,

(iii) and $\Lambda_{0}=\lambda_{0}$ if and only if $a(x) \equiv A(x)$ and $p_{i}(x) \equiv P_{i}(x)$ for $i=0$, $1, \ldots, k-1$.

Proof. The eigenvalues of the differential equations (1.1) and (1.2) are the reciprocals of the eigenvalue of the operators (3.4) and (3.5) respectively and conversely. From (4.1) and Lemma 3.2 it follows that $M$ and $N$ are $u_{0}$-positive. Lemma 3.7 guarantees the complete continuity of the operators (3.4) and (3.5) and thus Theorem 2.1 yields the conclusion (i). To verify (ii) we introduce the intermediate operator (3.6) and denote its largest positive eigenvalue by $\mu_{0}^{-1}$. By (4.1), (4.2) and Lemma 3.4 we have that $M \leqslant R$ with respect to the cone $P_{1}$. From Theorem 2.2 it follows that $\mu_{0} \leqslant \lambda_{0}$. Lemma 3.5 and (4.2) insure that $R \leqslant N$ with respect to the cone $P_{2}$. Applying Theorem 2.2 again we obtain $\Lambda_{0}$ $\leqslant \mu_{0}$ and hence conclusion (ii) follows.

The proof of the uniqueness condition (iii) involves introducing a sequence of intermediate operators $R_{0}, R_{1}, \ldots, R_{k-2}$ defined by

$$
R_{j}[u]=\int_{\alpha}^{\beta} G_{A}(x, s)\left[\sum_{i=0}^{j} P_{i}(s) u^{(i)}(s)+\sum_{i=j+1}^{k-1} p_{i}(s) u^{(i)}(s)\right] d s .
$$

If the reciprocals of the greatest positive eigenvalue of $R_{j}$ is denoted by $\Lambda_{k-1-j}$ we may obtain the ordering

$$
\Lambda_{0} \leqslant \Lambda_{1} \leqslant \Lambda_{2} \leqslant \cdots \leqslant \Lambda_{k-1} \leqslant \mu_{0} \leqslant \lambda_{0}
$$

by essentially the same arguments used above. From Theorem 2.1, the associated eigenfunctions of successive operators in this sequence are in the cone $P_{1}$ (or $P_{2}$ ) and from Theorem 2.2 are multiples of each other in the event that the successive eigenvalues are equal. Using the corresponding differential equations

$$
\begin{aligned}
{\left[A(x) u^{(k)}\right]^{(n-k)} } & =(-1)^{n-k} \Lambda_{k-1-i}\left(\sum_{i=0}^{\prime} P_{i}(x) u^{(i)}(x)+\sum_{i=j+1}^{k-1} p_{i}(x) u^{(i)}(x)\right), \\
u(\alpha) & =u^{\prime}(\alpha)=\cdots=u^{(k-1)}(\alpha)=0 \\
u_{1}(\beta) & =u_{1}^{\prime}(\beta)=\cdots=u_{1}^{(n-k-1)}(\beta)=0
\end{aligned}
$$


we can then derive the equality of the coefficient functions one at a time to reach conclusion (iii), and the theorem is proved.

By the same techniques the following theorems may be demonstrated. We omit the proofs.

THEOREM 4.2. If the coefficients of the differential equations (1.1) and (1.2) satisfy

$$
0 \leqslant P_{i}(x) \text { and } 0 \leqslant \int_{x}^{\beta} p_{i}(s) d s \leqslant \int_{x}^{\beta} P_{i}(s) d s
$$

on $[\alpha, \beta]$ for $i=0,1, \ldots, k-1$, and $\int_{\alpha}^{x}(1 / a(s)) d s \leqslant \int_{\alpha}^{x}(1 / A(s)) d s$ on $[\alpha, \beta]$, then $\Lambda_{0} \leqslant \lambda_{0}$ with equality if and only if $a(x) \equiv A(x)$ and $p_{i}(x) \equiv P_{i}(x), i=0$, $1, \ldots, k-1$.

THEOREM 4.3. If $0<A(x)<a(x)$ on $[\alpha, \beta]$ and $0 \leqslant \int_{x}^{\beta} p_{i}(s) d s<$ $\int_{x}^{\beta} P_{i}(s) d s$ on $[\alpha, \beta], i=0,1, \ldots, k-1$, then the least positive eigenvalues of the equations (1.1) and (1.2) satisfy $\Lambda_{0} \leqslant \lambda_{0}$ with equality if and only if $A(x)$ $\equiv a(x)$ and $P_{i}(x) \equiv p_{i}(x)$ for $i=0,1, \ldots, k-1$.

In [2] Z. Nehari used a special "Riccati System" to study the $(k, n-k)$ focal points of the equation $Y^{(n)}-(-1)^{n-k} P Y=0$. When this Riccati system is applied to equations of the form

$$
\left[a(x) u^{(k)}(x)\right]^{(n-k)}-(-1)^{n-k} \sum_{i=0}^{k-1} p_{i}(x) u^{(i)}(x)=0
$$

and

$$
\left[A(x) u^{(k)}(x)\right]^{(n-k)}-(-1)^{n-k} \sum_{i=0}^{k-1} P_{i}(x) u^{(i)}(x)=0,
$$

the following generalization of Nehari's Theorem 5.2 is obtained:

THEOREM 4.4 (NEHARI). Let $\eta_{k, n-k}(p)$ and $\eta_{k, n-k}(P)$ denote the $(k, n-$ $k$ )-focal points associated with the equations (4.5) and (4.6) respectively. Assume that

(i) $0<A(x) \leqslant a(x)$,

(ii) $0 \leqslant p_{i}(x) \leqslant P_{i}(x)$ for $i=0,1, \ldots, k-1$;

then $\eta_{k, n-k}(P)<\eta_{k, n-k}(p)$.

Corollary. If $0 \leqslant p_{i}(x), i=0,1, \ldots, k-1$, then the smallest positive eigenvalue $\lambda_{0}$ of the equation (1.1) is a monotone decreasing function of the length of the interval $[\alpha, \beta]$.

Proof. Let $\lambda_{0}\left(\beta_{i}\right)$ denote the smallest positive eigenvalue of (1.1) on the interval $\left[\alpha, \beta_{i}\right]$. We must demonstrate that $\beta_{1}<\beta_{2}$ implies $\lambda_{0}\left(\beta_{2}\right)<\lambda_{0}\left(\beta_{1}\right)$. We assume that this conclusion is false, i.e. $\lambda_{0}\left(\beta_{1}\right) \leqslant \lambda_{0}\left(\beta_{2}\right)$. Introducing the equations 


$$
\left[a(x) u^{(k)}(x)\right]^{(n-k)}-(-1)^{n-k} \sum_{i=0}^{k-1} \lambda_{0}\left(\beta_{1}\right) p_{i}(x) u^{(i)}(x)=0
$$

and

$$
\left[a(x) u^{(k)}(x)\right]^{(n-k)}-(-1)^{n-k} \sum_{i=0}^{k-1} \lambda_{0}\left(\beta_{2}\right) p_{i}(x) u^{(i)}(x)=0,
$$

we observe that $\beta_{1}$ and $\beta_{2}$ are the ( $\left.k, n-k\right)$-focal points of (4.7) and (4.8) respectively. Since $\lambda_{0}\left(\beta_{1}\right) p_{i}(x) \leqslant \lambda_{0}\left(\beta_{2}\right) p_{i}(x), i=0,1, \ldots, k-1$, it follows from Theorem 4.1 that $\beta_{2} \leqslant \beta_{1}$ which provides a contradiction and proves the corollary.

As an application of Theorem 4.1 we may extend the above Theorem of $Z$. Nehari by replacing the pointwise restrictions with integral inequalities.

THEOREM 4.5. Let $\eta_{k, n-k}(p)$ and $\eta_{k, n-k}(P)$ denote the $(k, n-k)$-focal points associated with the equations (4.5) and (4.6) respectively. Assume that

$$
0<p_{i}(x) \text { for } i=0,1,2, \ldots, k-1 \text {, }
$$

(ii) $\quad 0 \leqslant \int_{x}^{\beta_{1}} p_{i}(s) d s \leqslant \int_{x}^{\beta_{1}} P_{i}(s) d s$ for $i=0,1,2, \ldots, k-1$,

(iii) $\quad \int_{\alpha}^{x} \frac{1}{a(s)} d s \leqslant \int_{\alpha}^{x} \frac{1}{A(s)} d s$,

where $\beta_{1}=\eta_{k, n-k}(p)$, then $\eta_{k, n-k}(P) \leqslant \eta_{k, n-k}(p)$.

PROOF. Denote $\eta_{k, n-k}(p)$ and $\eta_{k, n-k}(P)$ by $\beta_{1}$ and $\beta_{2}$ respectively. Assuming the theorem is false we take $\beta_{1}<\beta_{2}$. Then the smallest positive eigenvalues of the problems (1.1) and (1.2) on the intervals $\left[\alpha, \beta_{1}\right]$ and $\left[\alpha, \beta_{2}\right]$ respectively are $\lambda_{0}\left(\beta_{1}\right)=1$ and $\Lambda_{0}\left(\beta_{2}\right)=1$. By the above corollary the smallest positive eigenvalue $\Lambda_{0}$ of (1.2) is monotone decreasing as a function of the end point $\beta$ of the interval $[\alpha, \beta]$. Hence $\beta_{1}<\beta_{2}$ implies that the smallest positive eigenvalue $\Lambda_{0}\left(\beta_{1}\right)$ of (1.2) over the interval $\left[\alpha, \beta_{1}\right]$ satisfies $\lambda_{0}\left(\beta_{1}\right)=1<$ $\Lambda_{0}\left(\beta_{1}\right)$. However, Theorem 4.1 with $\beta=\beta_{1}$ yields the inequality $\Lambda_{0}\left(\beta_{1}\right) \leqslant$ $\lambda_{0}\left(\beta_{1}\right)$, and thus the contradiction completes the proof.

Theorem 4.1 also allows us to investigate the relationship between the smallest positive eigenvalues of the $(k, n-k)$-focal point eigenvalue problem:

$$
\begin{gathered}
(-1)^{n-k}\left[a(x) u^{(k)}(x)\right]^{(n-k)}=\lambda p(x) u(x), \\
u(\alpha)=u^{\prime}(\alpha)=\cdots=u^{(k-1)}(\alpha)=0, \\
u_{1}(\beta)=u_{1}^{\prime}(\beta)=\cdots=u_{1}^{(n-k-1)}(\beta)=0,
\end{gathered}
$$


and that of its adjoint $(n-k, k)$-focal point eigenvalue problem:

$$
\begin{aligned}
(-1)^{n-k}\left[a(x) \nu^{(n-k)}(x)\right]^{(k)} & =\mu p(x) \nu(x), \\
\nu(\alpha)= & \nu^{\prime}(\alpha)=\cdots=\nu^{(n-k-1)}(\alpha)=0, \\
\nu_{1}(\beta)=\nu_{1}^{\prime}(\beta)=\cdots=\nu_{1}^{(k-1)}(\beta) & =0,
\end{aligned}
$$

where $u_{1}(x)$ and $\nu_{1}(x)$ are defined by

$$
u_{1}(x)=a(x) u^{(k)}(x) \text { and } \nu_{1}(x)=a(x) \nu^{(n-k)}(x) .
$$

If we assume that $p(x)>0$ on $[\alpha, \beta]$ then the existence of the smallest positive eigenvalues for both (4.9) and (4.10) follows from Theorem 4.1. It also follows that the associated unique (normalized) eigenfunctions are positive on $(\alpha, \beta]$. From the general theory of linear operators we know that the eigenvalues of two adjoint equations are complex conjugates of each other. Consequently, the real smallest positive eigenvalues of (4.9) and (4.10) are equal. This fact could be obtained directly from (4.9) and (4.10) since the eigenfunctions associated with the least eigenvalues $\lambda_{0}$ and $\mu_{0}$ are positive. Multiplication of (4.9) by $\nu$, subtracting the equation (4.10) multiplied by $u$ and integrating over $[\alpha, \beta]$ yields the identity

$$
0=\left(\lambda_{0}-\mu_{0}\right) \int_{\alpha}^{\beta} p(s) u(s) \nu(s) d s .
$$

Since $p(x)>0, u(x)>0, v(x)>0$ on $(\alpha, \beta)$ we have $\lambda_{0}=\mu_{0}$.

If an equation of the form

$$
\left[a(x) u^{(k)}(x)\right]^{(n-k)}-(-1)^{n-k} p(x) u(x)=0
$$

does not possess a $(k, n-k)$-focal point on $(\alpha, \beta)$ it is said to be $(k, n-k)$-disfocal on $[\alpha, \beta)$. From the corollary to Theorem 4.4 it easily follows that equation (4.11) is $(k, n-k)$-disfocal on $[\alpha, \beta)$ if and only if the smallest positive eigenvalue $\lambda_{0}$ of the eigenvalue problem (4.9) satisfies $\lambda_{0}>1$. Combining this with the above remarks concerning focal points of equation (4.11) and its adjoint equation,

$$
\left[a(x) u^{(n-k)}(x)\right]^{(k)}-(-1)^{k} p(x) u(x)=0,
$$

we are led to the following theorem.

THEOREM 4.6. Assume $p(x)>0$. Then the following statements are equivalent:

(A) The equation (4.11) is ( $k, n-k)$-disfocal on $[\alpha, \beta)$.

(B) The equation (4.12) is $(n-k, k)$-disfocal on $[\alpha, \beta)$.

(C) The least positive eigenvalue $\lambda_{0}$ of (4.9) satisfies $\lambda_{0}>1$. 
Finally we note that the general $n$th order differential equation

$$
y^{(n)}(x)+b_{n-1}(x) y^{(n-1)}(x)+\cdots+b_{0}(x) y(x)=0
$$

is equivalent to the equation

$$
\left[a(x) y^{(n-1)}(x)\right]^{\prime}+\sum_{i=0}^{n-2} p_{i}(x) y^{(i)}(x)=0
$$

where $a(x)=\exp \int_{\alpha}^{x} b_{n-1}(s) d s$ and $p_{i}(x)=a(x) b_{i}(x)$ for $i=0,1,2, \ldots, n-$ 2. Thus, it is possible to consider a $(n-1,1)$-focal point problem for the general $n$th order equation (1.3).

\section{REFERENCES}

1. M. A. Krasnosel'skir, Positive solutions of operator equations, Fizmatgiz, Moscow, 1962; English transl., Noordhoff, Groningen, 1964. MR 26 \#2862; 31 \#6107.

2. Z. Nehari, Nonlinear techniques for linear oscillation problems (to appear).

3. - Oscillation criteria for second-order linear differential equations, Trans. Amer. Math. Soc. 85 (1957), 428-445. MR 19, 415.

4. C. A. Swanson, Comparison and oscillation theory of linear differential equations, Academic Press, New York, 1968.

5. C. C. Travis, Comparison of eigenvalues for linear differential equations of order 2n, Trans. Amer. Math. Soc. 177 (1973), 363-374. MR 47 \#5356.

DEPARTMENT OF MATHEMATICS AND STATISTICS, UNIVERSITY OF GUELPH, GUELPH, ONTARIO, CANADA N1G 2W1

DEPARTMENT OF MATHEMATICS, UNIVERSITY OF TENNESSEE, KNOXVILLE, TENNESSEE 37916 